\title{
Iterative Reconstruction of Magnetic Induction using Lorentz Transmission Electron Tomography
}

\author{
C. Phatak ${ }^{\mathrm{a}, *}$, D. Gürsoy ${ }^{\mathrm{b}}$ \\ ${ }^{a}$ Materials Science Division, Argonne National Laboratory, 9700 S. Cass Avenue, Argonne, IL \\ 60439, USA \\ ${ }^{b}$ Advanced Photon Source, Argonne National Laboratory, Argonne, IL 60439, USA.
}

The submitted manuscript has been created by UChicago Argonne, LLC, Operator of Argonne National Laboratory ("Argonne"). Argonne, a U.S. Department of Energy Office of Science Laboratory, is operated under Contract No. DE-AC02-06CH11357. The U.S. Government retains for itself, and others acting on its behalf, a paid-up nonexclusive, irrevocable worldwide license in said article to reproduce, prepare derivative works, distribute copies to the public, and perform publicly and display publicly, by or on behalf of the Government. 


\title{
Iterative Reconstruction of Magnetic Induction using Lorentz Transmission Electron Tomography
}

\author{
C. Phatak ${ }^{\mathrm{a}, *}$, D. Gürsoy ${ }^{\mathrm{b}}$ \\ ${ }^{a}$ Materials Science Division, Argonne National Laboratory, Argonne, IL 60439, USA. \\ ${ }^{b}$ Advanced Photon Source, Argonne National Laboratory, Argonne, IL 60439, USA.
}

\begin{abstract}
Intense ongoing research on complex nanomagnetic structures requires a fundamental understanding of the 3D magnetization and the stray fields around the nano-objects. 3D visualization of such fields offers the best way to achieve this. Lorentz transmission electron microscopy provides a suitable combination of high resolution and ability to quantitatively visualize the magnetization vectors using phase retrieval methods. In this paper, we present a formalism to represent the magnetic phase shift of electrons as a Radon transform of the magnetic induction of the sample. Using this formalism, we then present the application of common tomographic methods particularly the iterative methods, to reconstruct the 3D components of the vector field. We present an analysis of the effect of missing wedge and the limited angular sampling as well as reconstruction of complex 3D magnetization in a nanowire using simulations.
\end{abstract}

Keywords: electron tomography, three dimensional vector fields, transmission electron microscopy, phase retrieval

\footnotetext{
${ }^{*}$ Corresponding author

Email address: cd@anl.gov (C. Phatak)
} 


\section{Introduction}

There is a growing interest in nanomagnetic structures that exhibit complex topological spin states. The research is both fundamental to understand the underlying spin textures and the physical phenomena as well as applied to ensure the stability of the state and ability to control it. For example, skyrmions exhibit three-dimensional (3D) variation of spin texture in chiral magnetic thin films that are stabilized by the Dzyalshinskii-Moriya interaction $[1,2]$. They are often present at specific points in arrays in an otherwise uniform magnetization pattern. They are topologically protected so that they cannot unwind or alter their magnetization continuously. As a result, they are being studied intensively for next generation information storage [3]. Towards achieving this understanding, there is a need to quantitatively map and visualize the entire 3D magnetization in such materials. Not only is $3 \mathrm{D}$ information necessary but also at a high spatial resolution. Lorentz transmission electron microscopy (LTEM) provides an ideal combination of high spatial resolution and ability to quantitatively map the magnetization in nanostructures $[4,5]$.

It has been shown experimentally that combining LTEM with tomographic methods, it is possible to reconstruct the 3D magnetic vector potential of a thin Permalloy ( $\mathrm{NiFe}$ ) islands [6]. The approach used acquisition of four tilt series about two mutually orthogonal axis in the plane of the sample. For each axis, two tilt series were acquired with the sample in as-is orientation and flipped upside down in order to separate the magnetic component of the phase shift from the electrostatic component. The magnetic 
phase shift tilt series data was then used for reconstructing the individual vector components using the vectorial filtered backprojection algorithm $[7,8]$. Although the reconstruction showed a correct representation of the spatial variation of the vector components, it suffered from the usual artifacts of limited tilt tomography, such as blurring, and streaking [9]. Additionally the resolution of the components along the $z$ direction is not the same as that in the $x$ and $y$ directions.

In other tomographic techniques such as X-ray computed tomography, the weighted (or filtered) backprojection method is typically not used or only used as a starting point for the $3 \mathrm{D}$ reconstruction. The initial estimate obtained using this method is then improved using various forms of iterative reconstruction methods such as algebraic reconstruction technique (ART) or simultaneous iterative reconstruction technique (SIRT) [10]. These methods rely on posing the reconstruction as an inverse problem and rely on solving a set of equations using linear algebra methods. In order to be able to do so, a projector matrix needs to be established that connects the projection data with the data to be reconstructed.

In this paper, we present a formalism to represent the magnetic phase shift data as a Radon transform of the magnetic induction components directly such that we are able to define a projector matrix. We will then introduce briefly the four basic reconstruction methods: (1) Weighted Backprojection Method (WBP), (2) Regridding reconstruction method (Gridrec), (3) ART, and (4) SIRT. These methods are commonly available in freeware and commercial softwares and are used for standard scalar tomography $[11,12]$. These methods are then be applied to the reconstruction of vector field components 
of a uniformly magnetized nanosphere since its 3D magnetic field is analytically known. We perform a detailed analysis of the effect of missing wedge, effect of limited angular sampling on the reconstructed components using the four methods. Finally we show the application of these methods to reconstruct the complex 3D magnetization in a magnetic nanowire with circular cross-section.

\section{Methods}

\subsection{Projection equations for $3 D$ vector field reconstruction}

In order to perform 3D reconstruction of the vector field components using various algorithms including iterative methods, it is essential to establish the correct projection equations i.e. forward projection and backward projection for the magnetic phase shift of electrons in terms of the components of magnetic induction of the sample. The magnetic phase shift of the electrons in the presence of a vector potential $\mathbf{A}$, of the sample can be written from the Aharonov-Bohm relation as [13]:

$$
\phi_{m}\left(\mathbf{r}_{\perp}\right)=-\frac{e}{\hbar} \int \mathbf{A}(\mathbf{r}) \cdot \mathrm{d} \mathbf{l}
$$

where $\mathbf{r}_{\perp}$ represents the $2 \mathrm{D}$ position vector in the projection plane, which is perpendicular to the direction of projection given by $\mathbf{l}$, and $\mathbf{A}(\mathbf{r})$ represents the vector potential. Considering the direction of projection to be along $z$ axis, we have $\mathrm{d} \mathbf{l}=\mathrm{d} z \hat{\mathbf{e}}_{\mathbf{z}}$. Substituting this into the above equation and simplifying, we get:

$$
\phi_{m}\left(\mathbf{r}_{\perp}\right)=-\frac{e}{\hbar} \int A_{z}(\mathbf{r}) \mathrm{d} z
$$


70

Taking the derivative with respect to $y$, we get:

$$
\frac{\partial \phi_{m}}{\partial y}=-\frac{e}{\hbar} \int \frac{\partial A_{z}}{\partial y} \mathrm{~d} z
$$

${ }_{71}$ where $y$ is defined in the projection plane as $\mathbf{r}_{\perp}=x \hat{\mathbf{e}}_{\mathbf{x}}+y \hat{\mathbf{e}}_{\mathbf{y}}$. From the 72 equation of curl of $\mathbf{A}$, considering only the $x$ component of the curl, we have

$$
B_{x}=\frac{\partial A_{z}}{\partial y}-\frac{\partial A_{y}}{\partial z}
$$

73

Using equation (3) and equation (4), we get

$$
\begin{aligned}
\frac{\partial \phi_{m}}{\partial y} & =-\frac{e}{\hbar} \int\left(B_{x}+\frac{\partial A_{y}}{\partial z}\right) \mathrm{d} z \\
& =-\frac{e}{\hbar}\left(\int B_{x} \mathrm{~d} z-\int \frac{\partial A_{y}}{\partial z} \mathrm{~d} z\right)
\end{aligned}
$$

74

The second term of the above equation becomes zero, since the vector potential is assumed to be zero at infinity. Hence the equation relating the magnetic phase shift with the magnetic induction can be written as:

$$
\frac{\partial}{\partial y} \phi_{m}(x, y)=-\left(\frac{e}{\hbar}\right) \int_{-\infty}^{\infty} B_{x}(x, y, z) \mathrm{d} z
$$

Similar equation can be derived for the $x$ derivative of the magnetic phase shift as:

$$
\frac{\partial}{\partial x} \phi_{m}(x, y)=\left(\frac{e}{\hbar}\right) \int_{-\infty}^{\infty} B_{y}(x, y, z) \mathrm{d} z ;
$$

The above equations are similar to the projection equations of a scalar quantity. In fact they are the real space representation of the equations derived previously by Phatak et. al. [8]. Considering a tilt series around the $x$-axis, for a given value of $x$, in the $y-z$ plane, the vector component $B_{x}$ remains invariant as a function of the tilting of the sample by $\theta$ 
or equivalently tilting the beam by $-\theta$. Thus the projection along any line in the $(y, z)$ plane can be parametrized using the relation $(y(\rho, \xi), z(\rho, \xi))=$ $((\rho \cos \theta+\xi \sin \theta),(\rho \sin \theta-\xi \cos \theta))$. Here $\rho$ gives the distance of the line from the origin. This is shown schematically in Fig. 1. Equation (6) can now be rewritten for any tilt angle $\theta$ about the $x$-axis as:

$$
\frac{\partial}{\partial y} \phi_{m}(x, \rho, \theta)=-\left(\frac{e}{\hbar}\right) \int_{-\infty}^{\infty} B_{x}(x, \rho \cos \theta+\xi \sin \theta, \rho \sin \theta-\xi \cos \theta) \mathrm{d} \xi
$$

If we compare this equation with the definition of Radon transform:

$$
R(\theta, \rho)=\int_{-\infty}^{\infty} A(\rho \cos \theta-s \sin \theta, \rho \sin \theta+s \cos \theta) \mathrm{d} s,
$$

we can see that the Radon transform of a $2 \mathrm{D}$ slice of $B_{x}$ in $(y, z)$ plane for a given value of $x$, gives the respective sinogram at that $x$ in the projection array. The projections thus obtained are essentially the derivative of the magnetic phase shift with respect to $y$. This establishes the forward projection equation that can be used for computing the projections from the vector field component. The backward projection equations can then be simply computed using the inverse Radon transform as:

$$
B_{x}(x, y, z)=\int_{0}^{\pi} \nabla_{y} \phi_{m}(x, y \cos \theta+z \sin \theta, \theta) \mathrm{d} \theta .
$$

From henceforth, the gradient of the phase shift data is referred to as the projection data. Similar equation can be derived for the $y$ component of the magnetic induction using equation (7) and relating it to the tilt series about the $y$ axis as follows:

$$
B_{y}(x, y, z)=\int_{0}^{\pi} \nabla_{x} \phi_{m}(x \cos \gamma+z \sin \gamma, y, \gamma) \mathrm{d} \gamma
$$


where $\gamma$ is the tilt angle about the $y$ axis. The third component of the magnetic induction, $B_{z}$, can then be computed using the zero-divergence condition, $\nabla \cdot \mathbf{B}=0$, as:

$$
B_{z}(x, y, z)=-\int\left(\frac{\partial B_{x}}{\partial x}+\frac{\partial B_{y}}{\partial y}\right) \mathrm{d} z
$$

These relations can now be used in various reconstruction algorithms including iterative methods to reconstruct the 3D individual $x$ and $y$ components of the magnetic induction. Next we will briefly describe the various methods that will be compared in the paper for reconstructing the $x$ and $y$ components of the magnetic induction.

\subsection{Weighted Backprojection Method - WBP}

Weighted backprojection is one of the standard methods used in tomographic reconstruction of $3 \mathrm{D}$ datasets. The algorithm is derived by using the Fourier Slice Theorem [14] that relates the the measured projection data to the 2D Fourier transforms of the object cross section. According to Fourier Slice Theorem, the Fourier transform of each projection, samples a slice of the 2D Fourier space $F(u, v)$ of the original object, and the cross section of the object $f(x, y)$ can be recovered by a $2 \mathrm{D}$ simple inverse Fourier transform:

$$
f(x, y)=\int_{-\infty}^{\infty} \int_{-\infty}^{\infty} F(u, v) \exp [j 2 \pi(u x+v y)] d u d v .
$$

In polar coordinates $(w, \phi)$, equation (13) can be written as:

$$
f(x, y)=\int_{0}^{\pi} \int_{-\infty}^{\infty} F(w \cos \phi, w \sin \phi) \exp [j 2 \pi w t]|w| d w d \phi .
$$

where $t=x \cos \phi+y \sin \phi$. Since the Fourier slice method inherently acts like a blurring function due to oversampling of lower spatial frequencies as 
compared to higher spatial frequencies, a filtering operation (i.e., $|w|)$ is used to improve the reconstruction. Typically this filter is a ramp filter in Fourier space. However using only a ramp filter amplifies high frequency noise, therefore a ramp filter multiplied with a Hanning window filter is more preferred.

\subsection{Regridding Reconstruction Method - Gridrec}

The regridding reconstruction method (Gridrec) is a viable alternative to the standard WBP which is derived similarly by using the Fourier Slice Theorem [15]. In Gridrec, the Fourier transform of the measured projection data is first convolved with a convolution kernel, $W(u, v)$, and then the convolution output is evaluated at the points of a Cartesian grid. The corresponding convolution output $H(u, v)$ can be written as:

$$
H(u, v)=\int_{0}^{\pi} \int_{-\infty}^{\infty} F(w \cos \phi, w \sin \phi) W(u-w \cos \phi, v-w \sin \phi)|w| d w d \phi .
$$

In equation (13), the multiplication $F(w \cos \phi, w \sin \phi)|w|$ corresponds to the typical high-pass filtering operation in WBP. Then one can apply 2D FastFourier Transform (FFT) to obtain object cross section similar to equation (13). The convolution kernel $W(u, v)$ is usually selected such that it acts as a low-pass filter with cutoff frequencies corresponding to a few spatial grid spacings in a concentrated region of interest. Typically Gridrec allows up to a 20 -fold increase in computation speed compared to WBP due to the utilization of FFTs on a regular grid [16]. 


\subsection{Algebraic Reconstruction Technique - ART}

The second set of reconstruction methods formulate the problem in a linear algebra framework, thus attempting to iteratively solve a large linear system of equations of the form, $A x=b$, where $b$ is the projection data, $A$ is the projection operator, and $x$ is the unknown 3D object to be determined. The standard algebraic reconstruction technique also known as the Kaczmarz method [17] solves the set of equations in an iterative fashion. In this method, one iteration consists of updates to the solution for each angle using the following equation:

$$
x^{k+1}=x^{k}+\lambda_{k} \frac{b_{i}-a_{i} x^{k}}{\left\|a_{i}\right\|^{2}} a^{i},
$$

where $k$ is the iteration number, $i$ represents each row of matrix $b$ which in turn corresponds to a particular angle in the tilt series, and $\lambda_{k}$ is the relaxation parameter with a value between $(0,2)$. In the case of reconstruction of magnetic induction, $b$ is the projection data i.e., the gradient of the magnetic phase shift, $A$ is the projection/Radon operator and $x$ is either $B_{x}$ or $B_{y}$ depending on the tilt series. In typical implementation of this method, the relaxation parameter $\lambda_{k}$ is fixed to be 1 . However, the parameter can be adjusted or optimized for each iteration, that is, usually by progressively decreasing it with increased number of iterations. This usually improves the imaging quality, at the expense of convergence.

\subsection{Simultaneous Iterative Reconstruction Technique - SIRT}

A variation of the ART methods is the simultaneous iterative reconstruction technique in which the updates to the solution are computed by taking into account all the tilt angles simultaneously in one iteration. This can be 
written as:

$$
x^{k+1}=x^{k}+\lambda_{k} A^{T}\left(b-A x^{k}\right),
$$

Similar to the ART method, the relaxation parameter $\lambda_{k}$ has a value between $(0,2)$. The advantage of SIRT is that it typically results in higher contrastto-noise ratio in the reconstructions as compared to ART, and is more robust to outliers in measurement data.

In the presence of measurement errors, it is not trivial to make an accurate estimation on parameters for iterative algorithms (e.g., stopping criterion, relaxation parameter, etc.) that provides the best final image quality. Usually the difference between the measured and simulated projection data, or difference in image between consecutive iterations, can be used to obtain a general idea on the convergence behavior. Unfortunately in many cases this can be misleading as the solution can diverge from the true image, while the difference measures show convergence. Especially for the case of ART-like algorithms that update the images individually for each tilt angle, salt-and-pepper, or checkerboard pattern artifacts starts to build-up after some iterations. SIRT is more robust to similar artifacts due to the simultaneous updating strategy. In this work, we heuristically selected the stopping criteria that provided the best image quality for both algorithms.

\section{Results}

\subsection{Simulation for uniformly magnetized sphere}

The magnetic induction of a uniformly magnetized sphere can be calculated analytically and hence it serves as a good test object for evaluating the various methods for reconstruction of the 3D magnetic induction. 
The magnetic induction of a sphere of radius $a$ with the magnetization $\mathbf{M}=\left[M_{x}, M_{y}, M_{z}\right]$ is given as [18]:

$$
\left(\begin{array}{c}
B_{x} \\
B_{y} \\
B_{z}
\end{array}\right)=\left\{\begin{array}{cc}
\frac{2 \mu_{0}}{3}\left(\begin{array}{c}
M_{x} \\
M_{y} \\
M_{z}
\end{array}\right) & r \leq a \\
\frac{\mu_{0} a^{3}}{3 r^{5}}\left(\begin{array}{c}
3 x\left(y M_{y}+z M_{z}\right)+M_{x}\left(2 x^{2}-y^{2}-z^{2}\right) \\
3 y\left(x M_{x}+z M_{z}\right)-M_{y}\left(x^{2}-2 y^{2}+z^{2}\right) \\
3 z\left(x M_{x}+y M_{y}\right)-M_{z}\left(x^{2}+y^{2}-2 z^{2}\right)
\end{array}\right) & r>a
\end{array}\right.
$$

Similarly the magnetic phase shift of such a sphere can also be calculated analytically and can be written as a function of the tilt angle $\theta$ about the $x$ axis as [19]:

$$
\begin{aligned}
\phi_{m}(x, y, \theta) & =\left(\frac{e}{\hbar}\right) \frac{2 \mu_{0} a^{3}}{3} \times \\
& \left(\frac{x\left(M_{y} \cos \theta-M_{z} \sin \theta\right)-y M_{x}}{x^{2}+y^{2}}\right)\left\{\begin{array}{cc}
\left(1-\left(1-\frac{x^{2}+y^{2}}{a^{2}}\right)^{1.5}\right) & r \leq a \\
1 & r>a
\end{array}\right.
\end{aligned}
$$

A similar equation can be written for the magnetic phase shift as a function of tilt angle $\gamma$ about the $y$ axis. These equations were implemented in IDL programming language to compute the tilt series data. The unit vector of magnetization of the sphere was taken to be $[\cos (\pi / 6), \sin (\pi / 6), 0]$. Fig. 2(a) shows the phase shift computed for a tilt angle of $0^{\circ}$, and (b) shows the schematic of the projection data which is a function of $(x, y)$ co-ordinates of the projection plane and the tilt angle. Here the projection data consists of the individual components of the gradient of the phase shift. For e.g., 
the projection data from tilt series about $x$ axis consists of the $y$ component of the gradient of the phase shift and vice-versa for the tilt series about $y$ axis. Fig. 2(c) shows the 2D sections along plane 1 indicated in (b) for tilt series about $x$ axis and $y$ axis. The top row shows the gradient components computed from the phase shift (left side of equation (8) and the bottom row shows the forward projection computed from 3D $B_{x}$ and $B_{y}$ (right side of equation (8)) components respectively. Fig. 2(d) top row shows the projection data as a sinogram representation at $x=0$ for $x$ tilt series and $y=0$ for $y$ tilt series respectively (indicated by plane 2 in Fig. 2). Fig. 2(d) bottom row shows the sinogram computed using the Radon transform of the 3D magnetic induction along $x=0$ and $y=0$ of $B_{x}$ and $B_{y}$. All the images are scaled to the same intensity levels. Thus, Fig. 2(c) shows that the projection equations are valid for a given angle for all $(x, y)$ in the phase shift data, and Fig. 2(d) shows it is valid for all angles for a given $x$ or $y$ coordinate of the phase shift data.

Next we will test the reconstruction of magnetic induction components using the 4 different methods mentioned previously and analyze the various effects and limits of experimental tomography such as the effect of the missing wedge, and angular sampling of the tilt series. The reconstructions using WBP and SIRT methods were performed using the TomoJ plugin for ImageJ [12] and Gridrec and ART methods were performed using the TomoPy package [20]. The performance of the reconstruction methods was compared by computing the root mean square error (RMSE) separately inside and outside the object. This error was calculated in this manner because inside the object, the reconstructed vector field represents the magnetization of the ob- 
ject and outside represents the stray field from the object. Thus it helps in understanding and analyzing the error. The RMSE was calculated as follows:

$$
\boldsymbol{\mu}_{F} \equiv\left[\frac{1}{N_{D}} \sum_{i, j, k}(\bar{F}(i, j, k)-F(i, j, k))^{2} D(i, j, k)\right]^{1 / 2}
$$

where $F$ represents the individual reconstructed component, $\bar{F}$ represents the analytical value, $D(i, j, k)$ represents the region inside or outside the object, and $N_{D}$ represents the total number of pixels in the region $D(i, j, k)$.

\subsection{Effect of missing wedge}

In typical TEM tomography due to the limitation of the sample shape/size or the limitation of the instrument (holder, pole piece gap), an entire tilt series over $\pm 90^{\circ}$ is difficult to record. As a result, there is a missing wedge of information from the tilt range over which no data is recorded. The effect of this missing wedge is well established and well studied for scalar tomography in TEM such as HAADF-STEM tomography. Here we discuss the effect of the missing wedge of information on the vector field tomographic reconstruction. A projection tilt series data within the range of $\pm 80^{\circ}$ and $\pm 70^{\circ}$ with tilt steps of $2^{\circ}$ was used for reconstructing individual $B_{x}$ and $B_{y}$ components of the magnetic induction. The iterative reconstruction using ART was performed with 20 iterations while SIRT was performed with 40 iterations and a fixed relaxation parameter, $\lambda_{k}=1$. Fig. 3 shows the comparison of the reconstructed components for all the 4 methods. The reconstructed components are displayed 2D images along various planes. The orientation of the planes and the object is schematically shown in the top-right corner inset. All the images are scaled to the same intensity range indicated by the 
color bar. For each method, the top row shows the reconstruction for the tilt range of $\pm 80^{\circ}$ and the bottom row for the tilt range of $\pm 70^{\circ}$. As with scalar tomography, as the tilting range is reduced, the elongation artifacts can be observed. In scalar tomography such as reconstruction of shape or morphology of the object, the object is typically a discrete object in space. This is a major difference as compared to vector field tomography, wherein the components of vector field are not discrete i.e., each component varies continuously in and around the object except at the boundary of the object. As a result, even with only a slightly reduced tilt range of $\pm 80^{\circ}$, there is significant smearing of the components outside of the object. Fig. 4 shows a comparison of line plots of the $B_{x}$ component of the vector field along the white line indicated in Fig. 3 for all the 4 methods. It can be seen that the WBP method suffers the most from missing wedge artifacts, and the magnitude of the magnetization inside the nanosphere as well as the stray field outside varies significantly with reduced tilt range. Interestingly the Gridrec method suffers significantly less from the missing wedge of information particularly for magnetization inside the nanosphere, which does not show any effect of missing information. The stray field reconstructed using this method varies significantly as compared to the theoretical value. The reconstruction using ART shows the most promising result for reconstructing magnetization as well as stray fields with limited tilt range. However, the reconstructions have more noise as seen from the line plots. The reconstruction using SIRT reduces the noise significantly as compared to ART and shows the best reconstruction result for tilt range of $\pm 80^{\circ}$. However, the magnitude of the reconstructed components is slightly lower as compared to the theoretical 
value for the tilt range of $\pm 70^{\circ}$. This can be further improved by increasing the number of iterations. The reconstructed components outside the sphere (the stray field) show some directional influence. The reconstruction along the $x$ direction for $B_{x}\left(y\right.$ for $\left.B_{y}\right)$ is better than that along the $z$ direction. Table 1 shows the RMSE inside (subscript $i$ ) and outside (subscript $o$ ) the nanosphere for the reconstructed $x$ and $y$ components. The reconstructed $B_{z}$ component is not compared here since that component is not directly reconstructed using any of the tomographic reconstruction methods. However, it should be noted that since $B_{z}$ is computed using the $B_{x}$ and $B_{y}$ components, improved reconstruction of these components should directly also improve the reconstruction of the $B_{z}$ component.

Table 1: RMS errors in the reconstructed components as a function of limited tilt range.

\begin{tabular}{|c|c|c||c|c||c|c||c|c||}
\hline \multirow{2}{*}{} & \multicolumn{2}{|c||}{$B_{x, i}$} & \multicolumn{2}{c||}{$B_{x, o}$} & \multicolumn{2}{c||}{$B_{y, i}$} & \multicolumn{2}{c||}{$B_{y, o}$} \\
\cline { 2 - 8 } & $\pm 80^{\circ}$ & $\pm 70^{\circ}$ & $\pm 80^{\circ}$ & $\pm 70^{\circ}$ & $\pm 80^{\circ}$ & $\pm 70^{\circ}$ & $\pm 80^{\circ}$ & $\pm 70^{\circ}$ \\
\hline \hline WBP & 0.2228 & 0.2856 & 0.0262 & 0.0350 & 0.1541 & 0.1875 & 0.0174 & 0.0220 \\
Gridrec & 0.1668 & 0.1813 & 0.0274 & 0.0420 & 0.0926 & 0.1049 & 0.0160 & 0.0255 \\
ART & 0.1730 & 0.1815 & 0.0186 & 0.0246 & 0.0966 & 0.1051 & 0.0109 & 0.0146 \\
SIRT & 0.1105 & 0.1646 & 0.0169 & 0.0295 & 0.0747 & 0.1011 & 0.0105 & 0.0173 \\
\hline
\end{tabular}

\subsection{Effect of angular sampling}

The reconstruction of vector fields inherently requires at least two tilt series acquisitions to be performed to obtain two components. Additionally to separate the magnetic phase shift from the total phase shift, the sample has to be inverted and the tilt series need to be repeated. This can lead to extremely cumbersome experiments as well as large amounts of data to 
be generated. In order to reduce the data as well as experimental time, we have analyzed the effect of angular sampling during tilt series acquisition. By varying the tilt steps, the total number of images recorded can be reduced. For an ideal reconstruction, one would record images every $1^{\circ}$ or $0.5^{\circ}$ tilt step. Here we have tested the reconstruction of $B_{x}$ and $B_{y}$ components using the tilt series data within a range of \pm 72 with tilt steps of $4^{\circ}$ and $8^{\circ}$. Fig. 5 shows the comparison of the reconstructed components as compared to the theoretical values along various planes similar to Fig. 3. All the images are scaled to the same intensity range indicated by the color bar. For each method, the top row shows the reconstruction for a tilt step of $4^{\circ}$ and the bottom row for $8^{\circ}$. Fig. 6 shows a comparison of line plots of the $B_{x}$ component of the vector field along the white line indicated in Fig. 6 for all the 4 methods. As before, the WBP method suffers the most due to limited angular sampling. This also directly affects the resolution along $z$ axis. In spite of severe undersampling, the Gridrec method can be seen to reconstruct the magnetic field inside the nanosphere with very low error. This is clearly evident also from the line plots in Fig. 6. The reconstructions using ART and SIRT show similar variations in the magnetic field inside and outside the nanosphere and are able to reconstruct the values fairly close to the theoretical value. As anticipated, SIRT method shows less noise as compared to ART method. This shows that even with large under sampling of tilt images recorded every $8^{\circ}$, the magnetic field inside the object can be reconstructed very faithfully. But to reconstruct the stray fields (magnetic field outside the object), one has to sample at least every $4^{\circ}$ and use iterative methods such as ART/SIRT to obtain meaningful results. 


\subsection{Effect of the relaxation parameter}

As mentioned earlier, it is not trivial to estimate the parameters such as relaxation parameter, for iterative reconstruction methods. Here we have tested various values of the relaxation parameter on the SIRT method. The simulated phase shift data for the nanosphere with a tilt range of $\pm 72^{\circ}$ with tilt steps of $8^{\circ}$ was used to reconstruct the magnetic induction. Since these methods are computationally expensive, we have only studied the effect on the reconstruction of the $x$ component of the magnetic induction. The relaxation paramter was varied from 0.05 to 2.0 while keeping the number of iterations fixed. A successive error between two consecutive iterations was computed and plotted against the iteration number to give an idea about the convergence of the algorithm. Fig. 7(a) shows these plots for the various values of lambda. It can be seen that the error decreases at a much slower rate as the value of lambda is decreased. Fig. 7(b) shows the lineplot of the $x$ component of the vector field as shown previously. It is interesting to note that with fixed number of iterations, varying the value of lambda does not make significant difference on the reconstructed component of magnetization. This is also evident from the RMS values which change from 0.033 for $\lambda=2.0$ to 0.034 for $\lambda=0.05$. This shows that the relaxation parameter does not have a significant effect on the reconstructed components. However, from the error decay, a value of $\lambda=1.0$ can be seen to be an optimal value for reconstructions. It should be noted here however, that the projection images used for the SIRT reconstruction are simulated images and do not have significant noise as compared to typical experimental images. 


\subsection{Domain wall in nanowires}

From the previous section, it can be concluded that iterative methods can significantly improve the reconstruction of magnetic field for limited tilt range as well as under sampling. In particular, the magnetic field inside the object can be reconstructed with very low error margin. Finally to demonstrate the applicability of these methods to more complex magnetization patterns, we performed simulations to reconstruct the complex 3D magnetization inside a magnetic nanowire. In nanowires with circular cross section, there are two types of domain walls that can exist: (1) Transverse domain walls, and (2) Bloch point domain walls $[21,22]$. Both these type of domain walls have a complex magnetization pattern in 3D. Of particular interest are the Bloch point walls as they are topologically protected, i.e. they are stable and cannot deform under external perturbations. This is of significance for 3D magnetic domain wall memory applications [23, 24].

We performed micromagnetic simulations using OOMMF to obtain the complex magnetization pattern in such a nanowire. Fig. 8(a) shows the schematic of the magnetization inside the nanowire and location of the domain wall. The magnetization thus obtained was used for computation of the magnetic phase shift using a modified Mansuripur algorithm [25]. The phase shift was computed as a function of varying angle of the incident electron beam to simulate the tilt series about $x$ and $y$ axes. Fig. 8(b) shows the magnetic phase shift computed for $0^{\circ}$ tilt, (c) and (d) show the corresponding simulated in-focus and under-focus Lorentz TEM images. The location of the domain wall in the under focus image is indicated by the white arrow. The tilt series data was simulated for a tilt range of $\pm 70^{\circ}$ with tilt steps of 
$2^{\circ}$. The reconstructions were then performed using all the 4 methods. Fig. 9 shows a comparison of the reconstructed components in the $x-y$ plane for the four methods. The orientation of the nanowire and the planes is shown in the schematic. From the images, it is clear that there is significant error in the reconstructed components of the stray field (outside the nanowire). However, the reconstructed magnetization inside the nanowire matches very closely with that obtained from the micromagnetic simulations (indicated as theo). The $B_{z}$ component was computed using the divergenceless condition as described previously in equation (12). It can be seen that with the improvement of reconstruction of $B_{x}$ and $B_{y}$ components particularly by the Gridrec method, the reconstruction of $B_{z}$ component is also improved. Fig. 10 shows the $3 \mathrm{D}$ vector field plots of the magnetization inside the nanowire. The data is clipped along $y=0$ to show the circular rotation of the magnetization in 3D. Overall all the methods give better results as compared to WBP. Particularly the results obtained using Gridrec method show the best reconstruction of magnetization inside the nanowire. This demonstrates that with improved reconstruction methods, the complex 3D magnetization in nanoobjects such as nanowires can be faithfully reconstructed.

\section{Conclusions}

We have shown in this work, that the projection data for magnetic vector field tomography can be reformulated in terms of Radon projection of the magnetic induction of a nanostructure. This allows for implementing the reconstruction as a linear algebra problem such that various iterative algebraic reconstruction methods can be used. We have demonstrated the 
application of 4 common methods to reconstruct the vector field components of the magnetic induction of a uniformly magnetized nanosphere. From the analysis reconstructions for missing wedge and limited angular sampling, we can conclude that the improved methods such as Gridrec and SIRT perform better with lower errors that others. Overall, it was concluded that the iterative methods are better at reconstructing the magnetization inside the nano-object as compared to the stray field outside the object. This was demonstrated by reconstructing a more complex magnetization pattern around a domain wall in a magnetic nanowire.

However, these methods only reconstruct and improve the individual $x$ and $y$ components of the magnetic induction. Recent work by De Graef et. al. [26] has shown that the vector field reconstruction can be formulated in a model based iterative reconstruction method, where all the three components of magnetic induction can be reconstructed simultaneously. Additionally, the methods presented in this paper do not use any prior information or enforce any regularization conditions. Future work along this direction is in progress to incorporate smoothness criterion and couple the reconstructions to further improve the results.

\section{Acknowledgments}

Work by C.P was supported by the U.S. Department of Energy, Office of Science, Materials Sciences and Engineering Division. This work is partially supported by the U.S. Department of Energy under Contract No. DE-AC02$06 \mathrm{CH} 11357$. 
[1] P. Milde, D. Köhler, J. Seidel, L. M. Eng, A. Bauer, A. Chacon, J. Kindervater, S. Mühlbauer, C. Pfleiderer, S. Buhrandt, C. Schütte, A. Rosch, Unwinding of a skyrmion lattice by magnetic monopoles., Science (New York, N.Y.) 340 (6136) (2013) 1076-80. doi:10.1126/science.1234657.

URL http://www.ncbi.nlm.nih.gov/pubmed/23723232

[2] J. Sampaio, V. Cros, S. Rohart, A. Thiaville, A. Fert, Nucleation, stability and current-induced motion of isolated magnetic skyrmions in nanostructures., Nature nanotechnology 8 (11) (2013) 839-44. doi:10.1038/nnano.2013.210.

URL http://www.ncbi.nlm.nih.gov/pubmed/24162000

[3] N. S. Kiselev, A. N. Bogdanov, R. Schäfer, U. K. Röß ler, Chiral skyrmions in thin magnetic films: new objects for magnetic storage technologies?, Journal of Physics D: Applied Physics 44 (39) (2011) 392001. doi:10.1088/0022-3727/44/39/392001.

URL http: //stacks. iop.org/0022-3727/44/i=39/a=392001?key=crossref . d8b4b51d66d

[4] C. Phatak, A. Petford-Long, O. Heinonen, M. Tanase, M. De Graef, Nanoscale structure of the magnetic induction at monopole defects in artificial spin-ice lattices, Physical Review B 83 (17) (2011) 174431. doi:10.1103/PhysRevB.83.174431.

URL http://link.aps.org/doi/10.1103/PhysRevB .83.174431

[5] C. Phatak, Y. Liu, E. B. Gulsoy, D. Schmidt, E. Franke-Schubert, A. Petford-Long, Visualization of the magnetic structure of sculpted 
three-dimensional cobalt nanospirals., Nano letters 14 (2) (2014) 75964. doi:10.1021/nl404071u.

URL http://www.ncbi.nlm.nih.gov/pubmed/24444002

[6] C. Phatak, A. Petford-Long, M. De Graef, Three-Dimensional Study of the Vector Potential of Magnetic Structures, Physical Review Letters 104 (2010) 253901. doi:10.1103/PhysRevLett.104.253901.

URL http://link.aps.org/doi/10.1103/PhysRevLett.104.253901

[7] S. Lade, D. Paganin, M. Morgan, Electron tomography of electromagnetic fields, potentials and sources, Optics Communications 253 (4-6) (2005) 392-400. doi:10.1016/j.optcom.2005.04.071.

URL http://linkinghub.elsevier.com/retrieve/pii/S0030401805004190

[8] C. Phatak, M. Beleggia, M. De Graef, Vector field electron tomography of magnetic materials: theoretical development., Ultramicroscopy 108 (6) (2008) 503-13. doi:10.1016/j.ultramic.2007.08.002.

URL http://www.ncbi.nlm.nih.gov/pubmed/17804165

[9] I. Arslan, J. R. Tong, P. A. Midgley, Reducing the missing wedge: Highresolution dual axis tomography of inorganic materials, Ultramicroscopy 106 (2006) 994-1000. doi:DOI 10.1016/j.ultramic.2006.05.010.

[10] F. Natterer, The Mathematics of Computerized Tomography, SIAM Publications, 2001.

[11] P. C. Hansen, M. Saxild-Hansen, AIR Tools - A MATLAB package of algebraic iterative reconstruction methods, Journal of Computational and Applied Mathematics 236 (8) (2012) 2167-2178. 
doi:10.1016/j.cam.2011.09.039.

URL http: //linkinghub.elsevier.com/retrieve/pii/S0377042711005188

[12] C. Messaoudii, T. Boudier, C. O. Sanchez Sorzano, S. Marco, TomoJ: tomography software for three-dimensional reconstruction in transmission electron microscopy., BMC bioinformatics 8 (2007) 288. doi:10.1186/1471-2105-8-288.

URL http://www.pubmedcentral.nih.gov/articlerender.fcgi?artid=1976622\&tool=pm

[13] Y. Aharonov, D. Bohm, Significance of electromagnetic potentials in the quantum theory, Phys. Rev. 115 (3) (1959) 485-491.

[14] A. Kak, M. Slaney, Principles of Computerized Tomographic Imaging, SIAM Publications, 2001.

[15] B. A. Dowd, G. H. Campbell, R. B. Marr, V. Nagarkar, S. Tipnis, L. Axe, D. P. Siddons, Developments in synchrotron x-ray computed microtomography at the National Synchrotron Light Source, Proceedings of SPIE X-Ray Tomography II 3772 (1999) 224-236.

[16] F. Marone, M. Stampanoni, Regridding reconstruction algorithm for real-time tomographic imaging., Journal of synchrotron radiation 19 (Pt 6) (2012) 1029-37. doi:10.1107/S0909049512032864. URL http: //www.pubmedcentral.nih.gov/articlerender.fcgi?artid=3480277\&tool=pm

[17] S. Kaczmarz, Angenäherte auflösung von systemen linearer gleichungen, Bulletin International de l'Academie Polonaise des Sciences et des Lettres 35 (1937) 355-357. 
[18] J.D.Jackson, Classical Electrodynamics, John Wiley \& Sons, 1999.

[19] M. D. Graef, N. Nuhfer, M. McCartney, Phase contrast of spherical magnetic particles, Journal of Microscopy 194 (1) (1999) 84.

[20] D. Gursoy, F. D. Carlo, X. Xiao, C. Jacobsen, TomoPy : A framework for the analysis of synchrotron tomographic data, Journal of synchrotron radiation 21 .

[21] N. Biziere, C. Gatel, R. Lassalle-Balier, M. C. Clochard, J. E. Wegrowe, E. Snoeck, Imaging the fine structure of a magnetic domain wall in a $\mathrm{Ni}$ nanocylinder., Nano letters 13 (5) (2013) 2053-7. doi:10.1021/nl400317j. URL http://www.pubmedcentral.nih.gov/articlerender.fcgi?artid=3650658\&tool=pm

[22] S. Da Col, S. Jamet, N. Rougemaille, A. Locatelli, T. O. Mentes, B. S. Burgos, R. Afid, M. Darques, L. Cagnon, J. C. Toussaint, O. Fruchart, Observation of Bloch-point domain walls in cylindrical magnetic nanowires, Physical Review B 89 (18) (2014) 180405. doi:10.1103/PhysRevB.89.180405. URL http://link.aps.org/doi/10.1103/PhysRevB.89.180405

[23] D. A. Allwood, G. Xiong, C. C. Faulkner, D. Atkinson, D. Petit, R. P. Cowburn, Magnetic domain-wall logic., Science (New York, N.Y.) 309 (5741) (2005) 1688-92. doi:10.1126/science.1108813.

URL http://www.ncbi.nlm.nih.gov/pubmed/16151002

[24] S. S. P. Parkin, M. Hayashi, L. Thomas, Magnetic domain-wall racetrack memory., Science (New York, N.Y.) 320 (5873) (2008) 190-4. 
doi:10.1126/science.1145799.

URL http://www.ncbi.nlm.nih.gov/pubmed/18403702

[25] M. Mansuripur, Computation of electron diffraction patterns in Lorentz electron microscopy of thin magnetic films, Journal of applied physics 69 (4) (1991) 2455-2464.

URL http : //ieeexplore. ieee.org/xpls/abs_all $\cdot$ jsp?arnumber $=5139748$

[26] E. Humphrey, M. De Graef, On the computation of the magnetic phase shift for magnetic nano-particles of arbitrary shape using a spherical projection model., Ultramicroscopy 129 (2013) 36-41. doi:10.1016/j.ultramic.2013.03.006.

URL http://www.ncbi.nlm.nih.gov/pubmed/23598072 


\section{Figures}

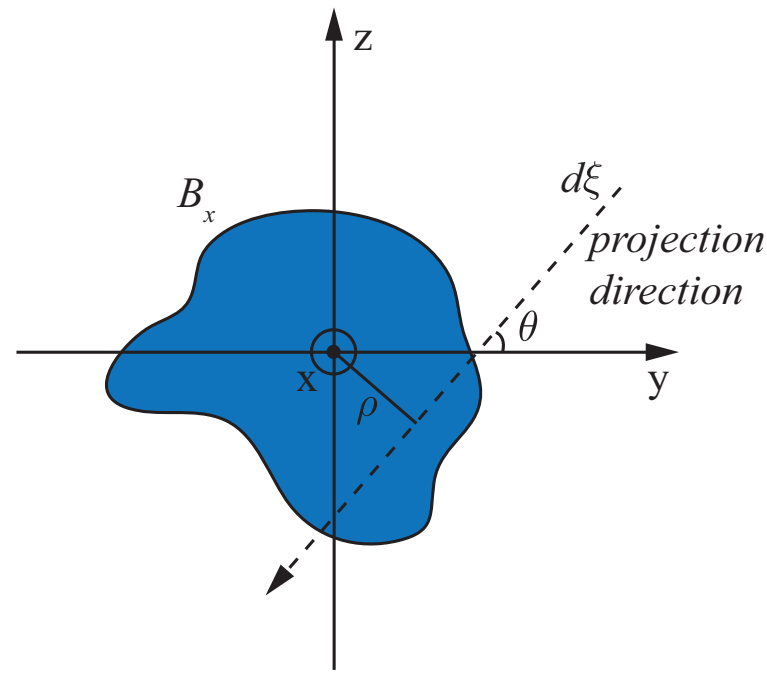

Figure 1: Schematic figure showing the orientation of projection geometry. The figure shows the $y-z$ plane at $x=0$ and the projection direction $\mathrm{d} \xi$ at an angle of $\theta$ and a distance $\rho$ from the origin. 


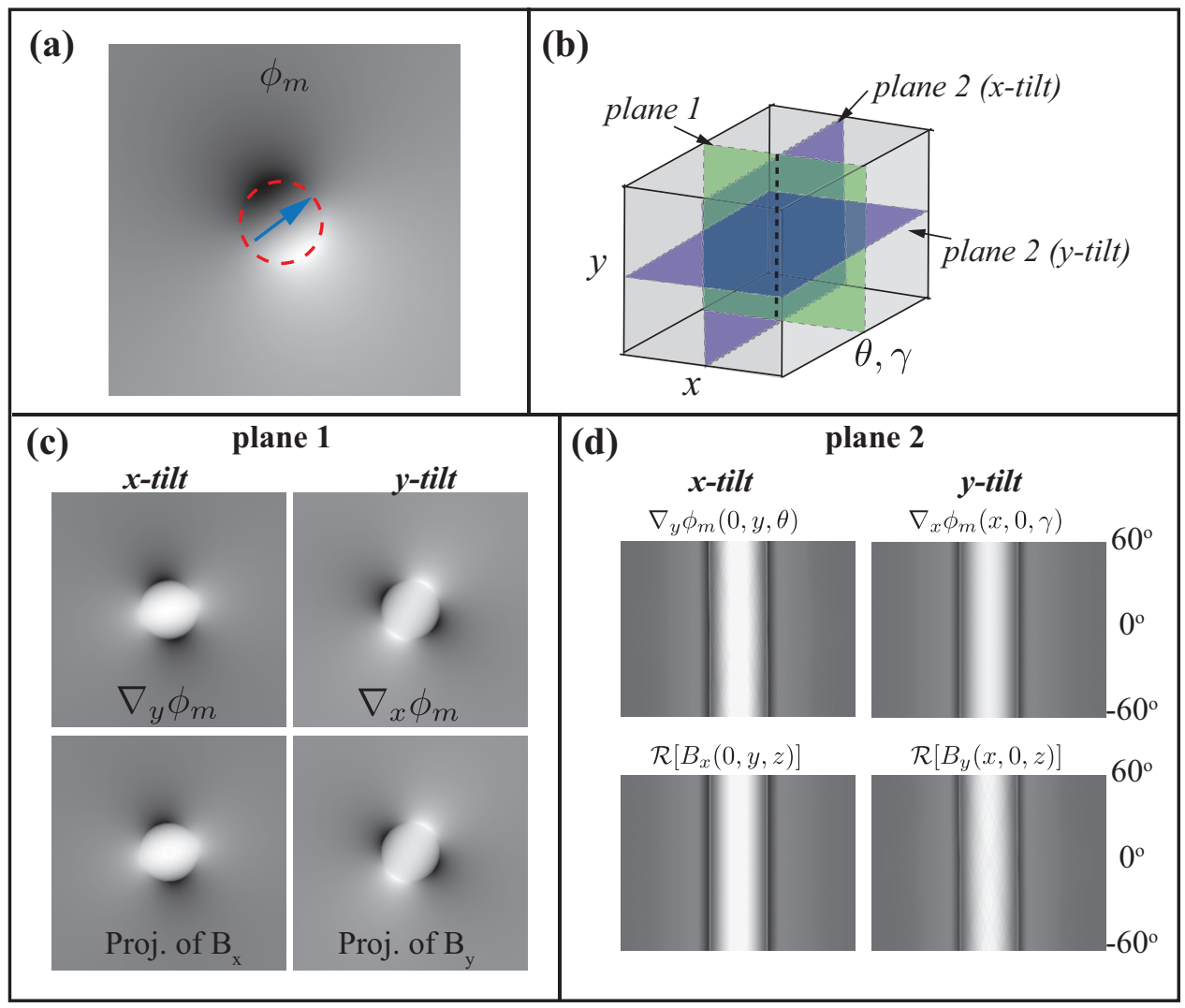

Figure 2: (a) Phase shift of a uniformly magnetized sphere (outline indicated by dashed red line) at $0^{\circ}$ tilt with the magnetization direction indicated by the blue arrow, (b) schematic representation of the projection data cube, (c) Projection data along plane 1 obtained using gradient of phase shift (top row) and integration of 3D magnetic induction components (bottom row), and (d) Projection data along plane 2 represented as sinograms obtained using gradient of phase shift (top row) and Radon transform of 3D magnetic induction components (bottom row). 


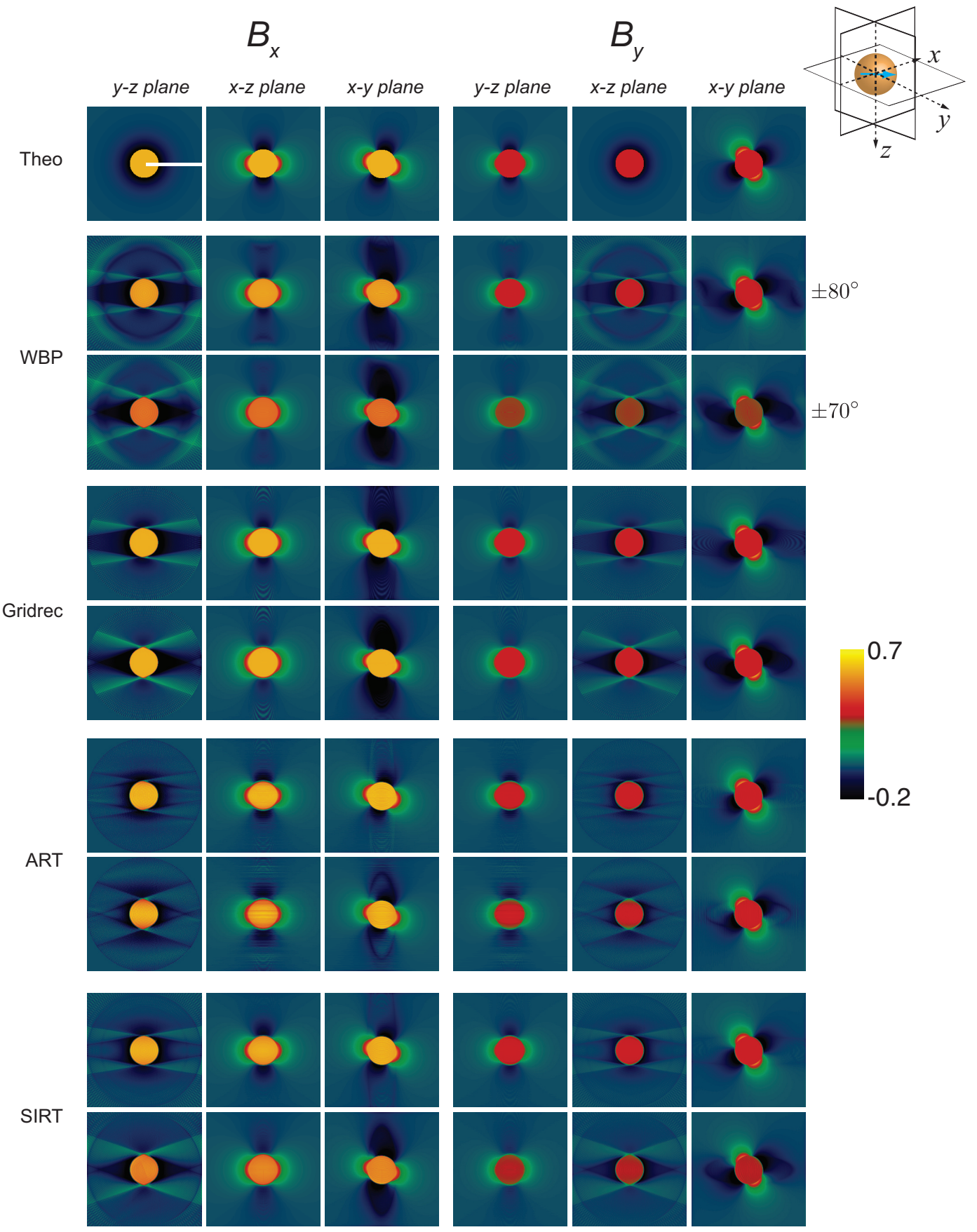

Figure 3: Comparison of reconstructed components of the vector field shown as 2D images along various planes. The orientation of the planes is indicated in the schematic. For each method, the top row shows reconstruction obtained using a tilt range of $\pm 80^{\circ}$ and the bottom row using $\pm 70^{\circ}$. All the images are scaled to the same intensity range indicated by the color bar. 

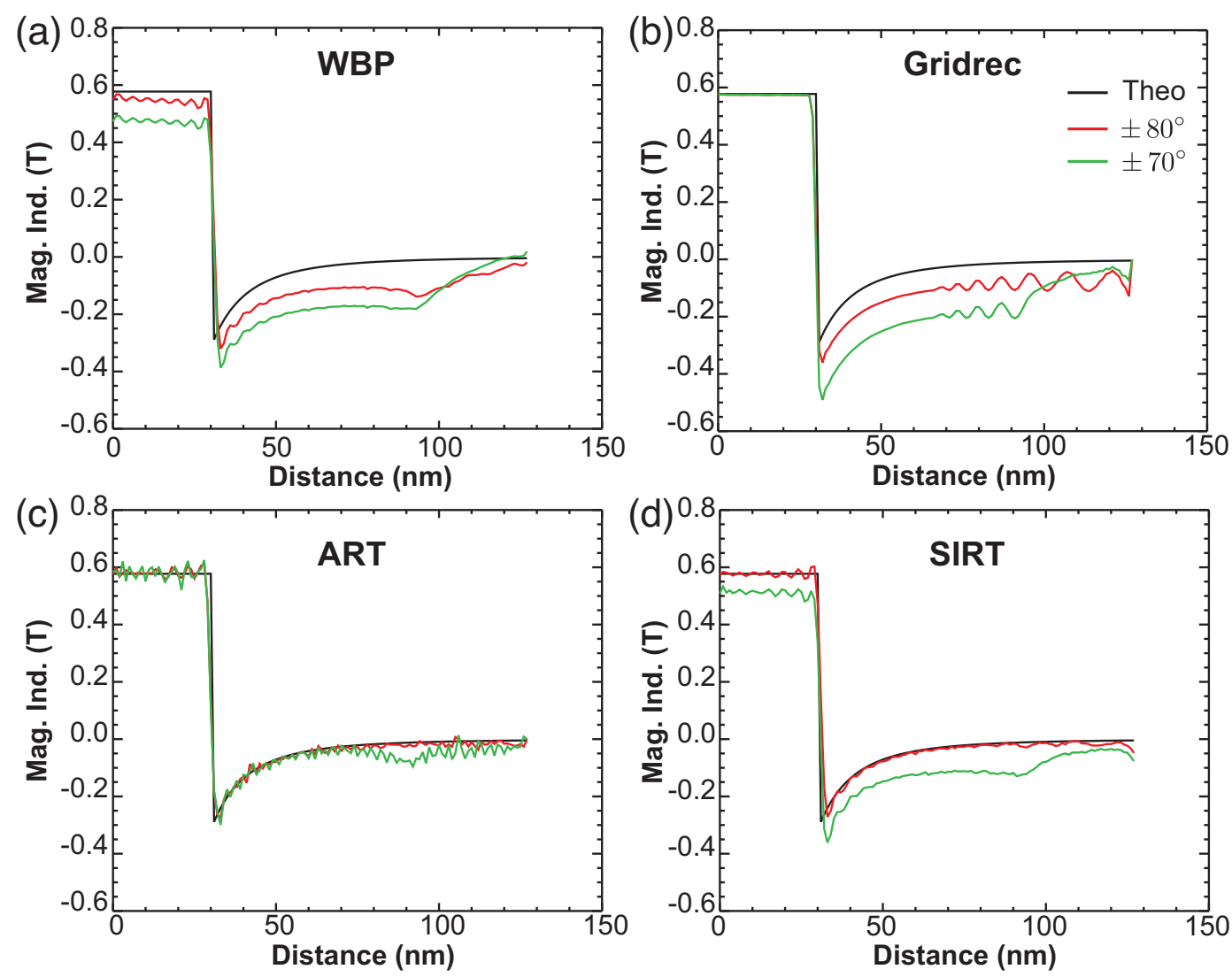

Figure 4: Comparison of line plots of the reconstructed $B_{x}$ component along the direction indicated as a white line in Fig. 3. 


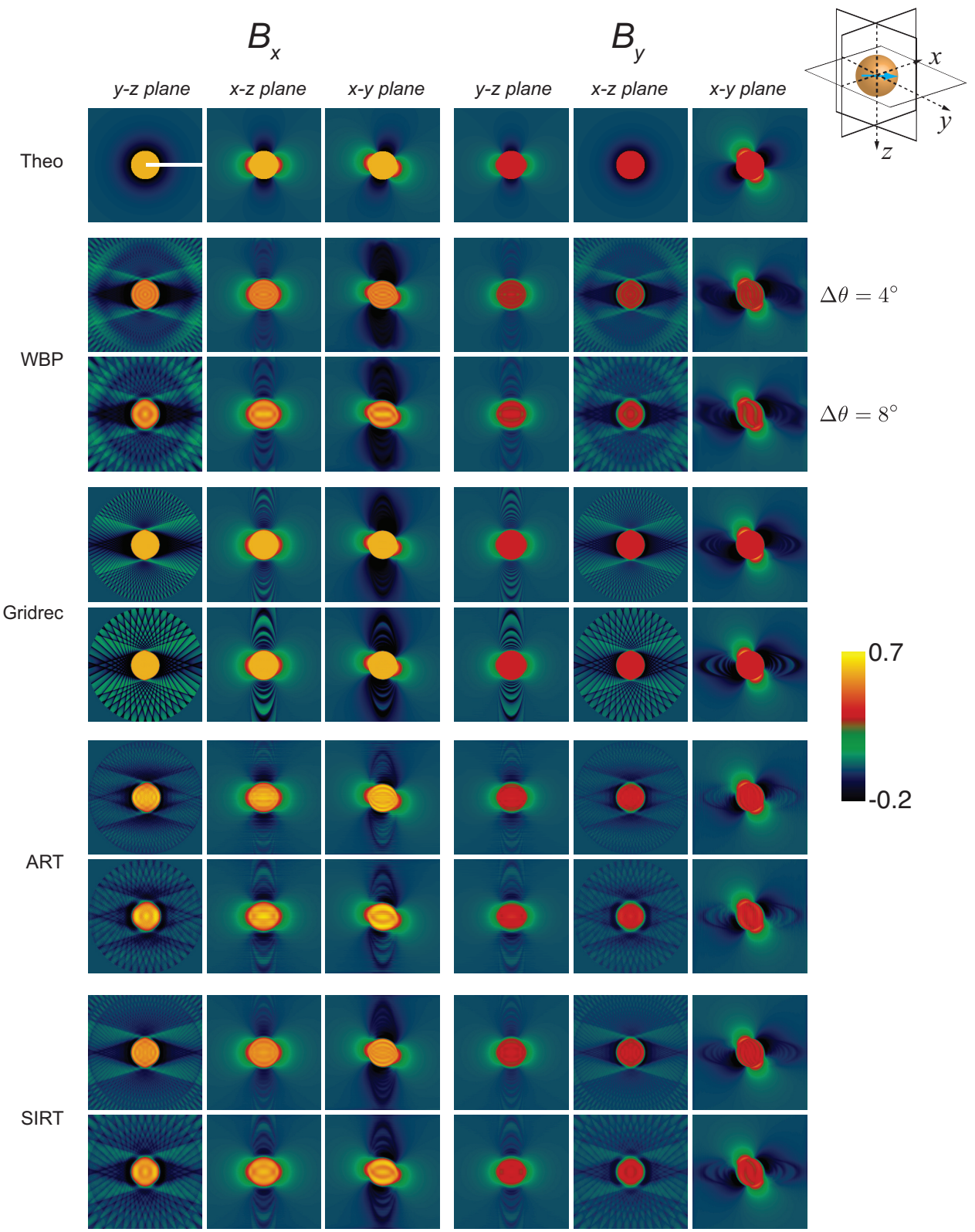

Figure 5: Comparison of reconstructed components of the vector field shown as 2D images along various planes. The orientation of the planes is indicated in the schematic. For each method, the top row shows reconstruction obtained using a tilt step of $4^{\circ}$ and the bottom row using $8^{\circ}$. All the images are scaled to the same intensity range indicated by the color bar. 

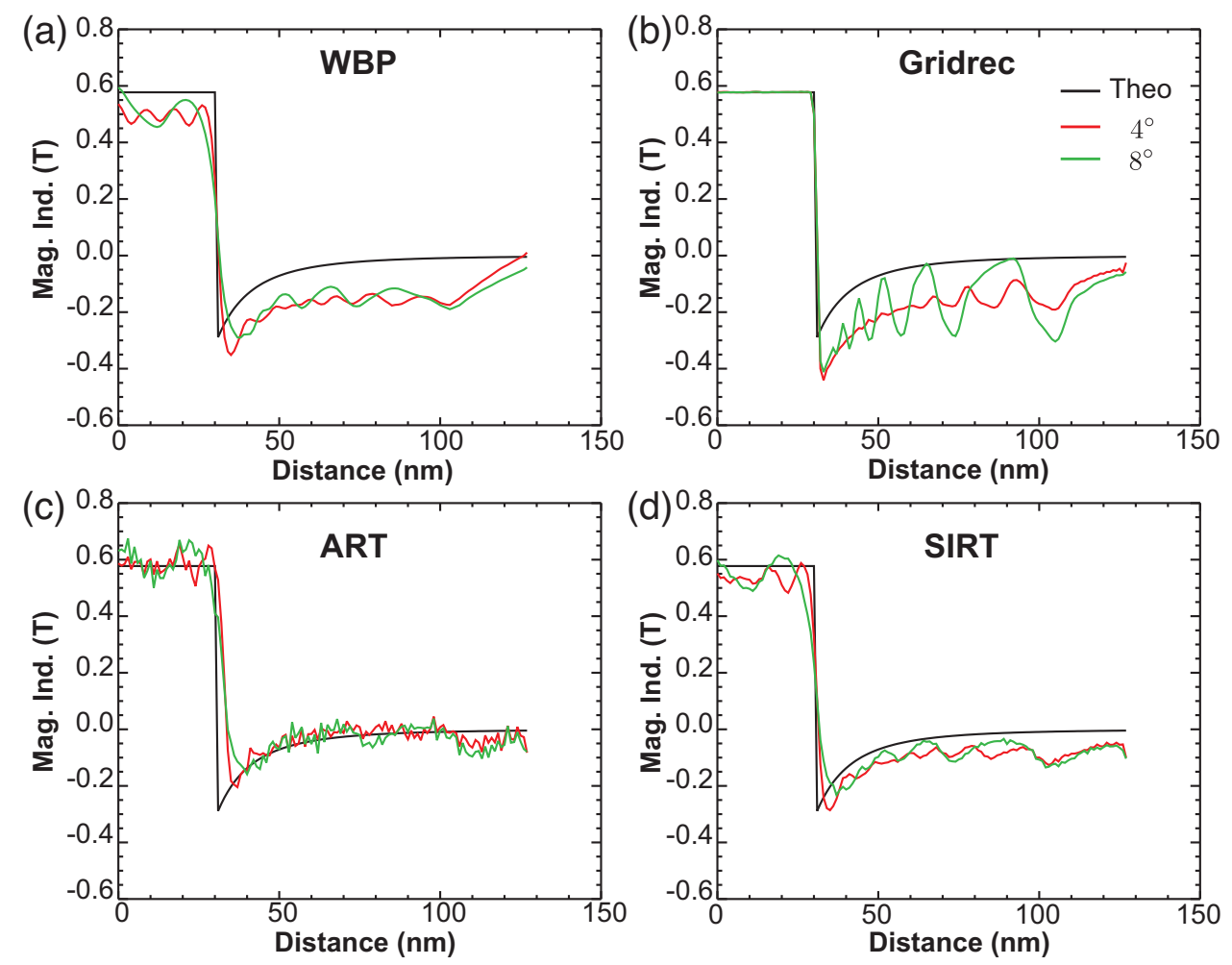

Figure 6: Comparison of line plots of the reconstructed $B_{x}$ component along the direction indicated as a white line in Fig. 5. 
(a)

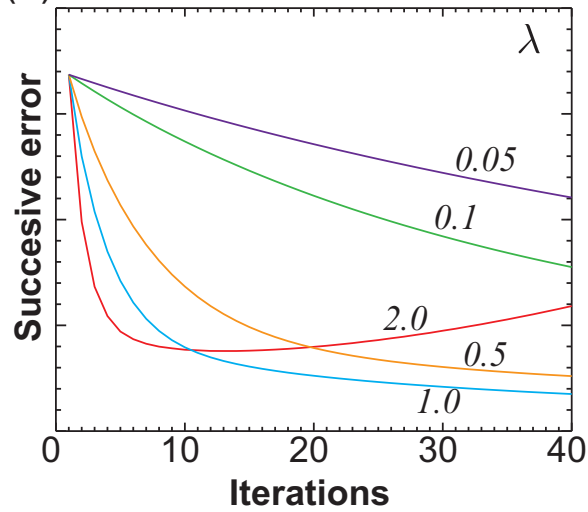

(b)

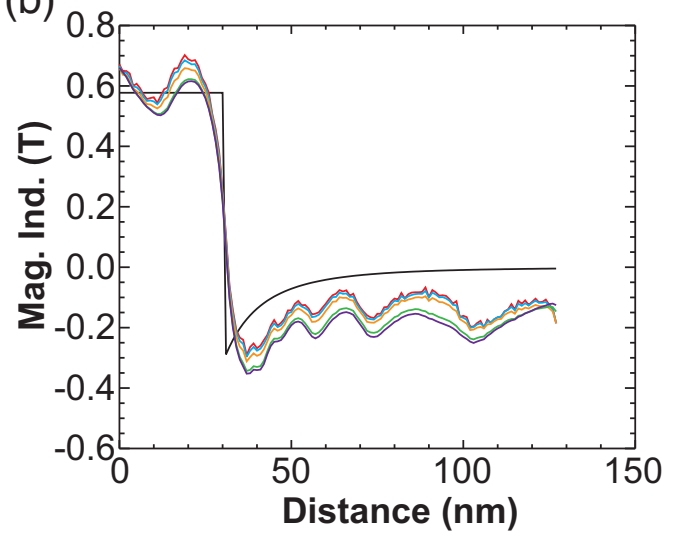

Figure 7: (a) Plot showing the decay of error as a function of iteration number for SIRT method for varying values of the relaxation parameter, $\lambda$, and (b) comparison of line plots of the reconstructed $B_{x}$ component along the direction indicated in Fig. 5. 
(a)

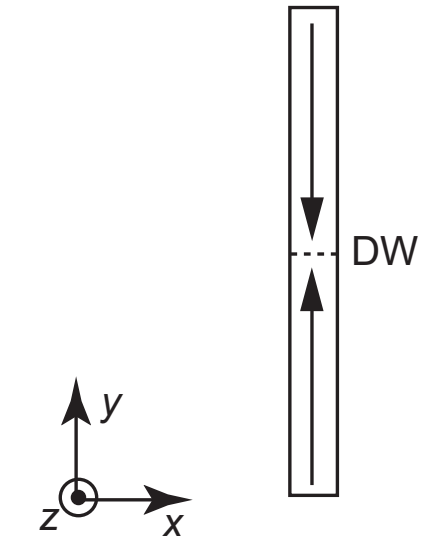

(c)

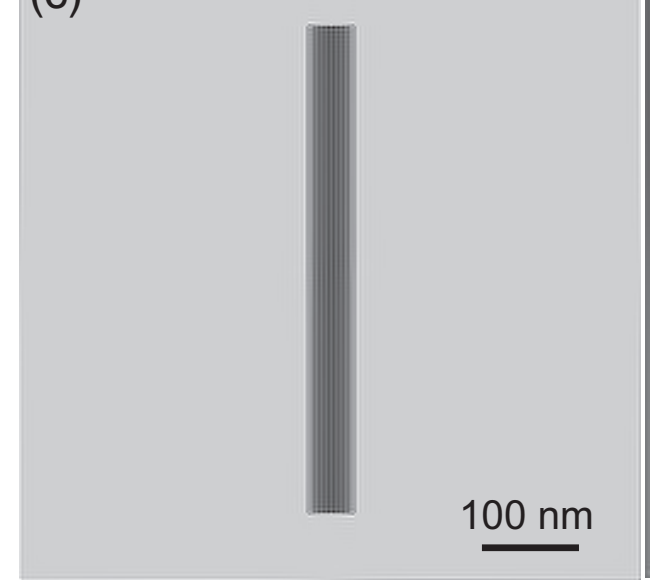

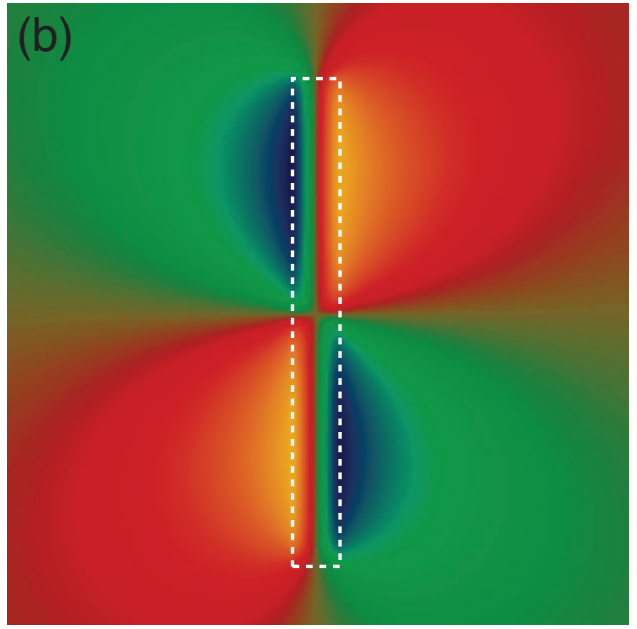

(d)

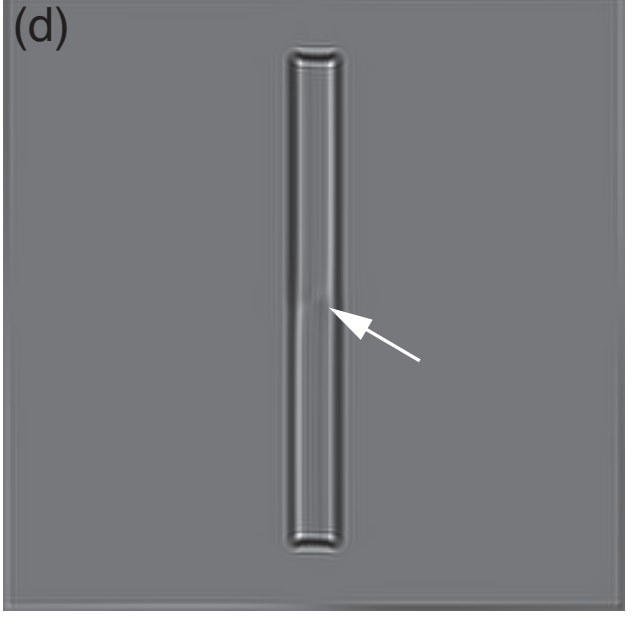

Figure 8: (a) Schematic showing the magnetization setup in the nanowire and the location of the domain wall (DW), (b) shows the simulated magnetic phase shift from the magnetization obtained using micromagnetic simulations at $0^{\circ}$ tilt, (c ) and (d) show the simulated in-focus and under-focus LTEM images. The presence of domain wall is indicated by the white arrow in (d). 


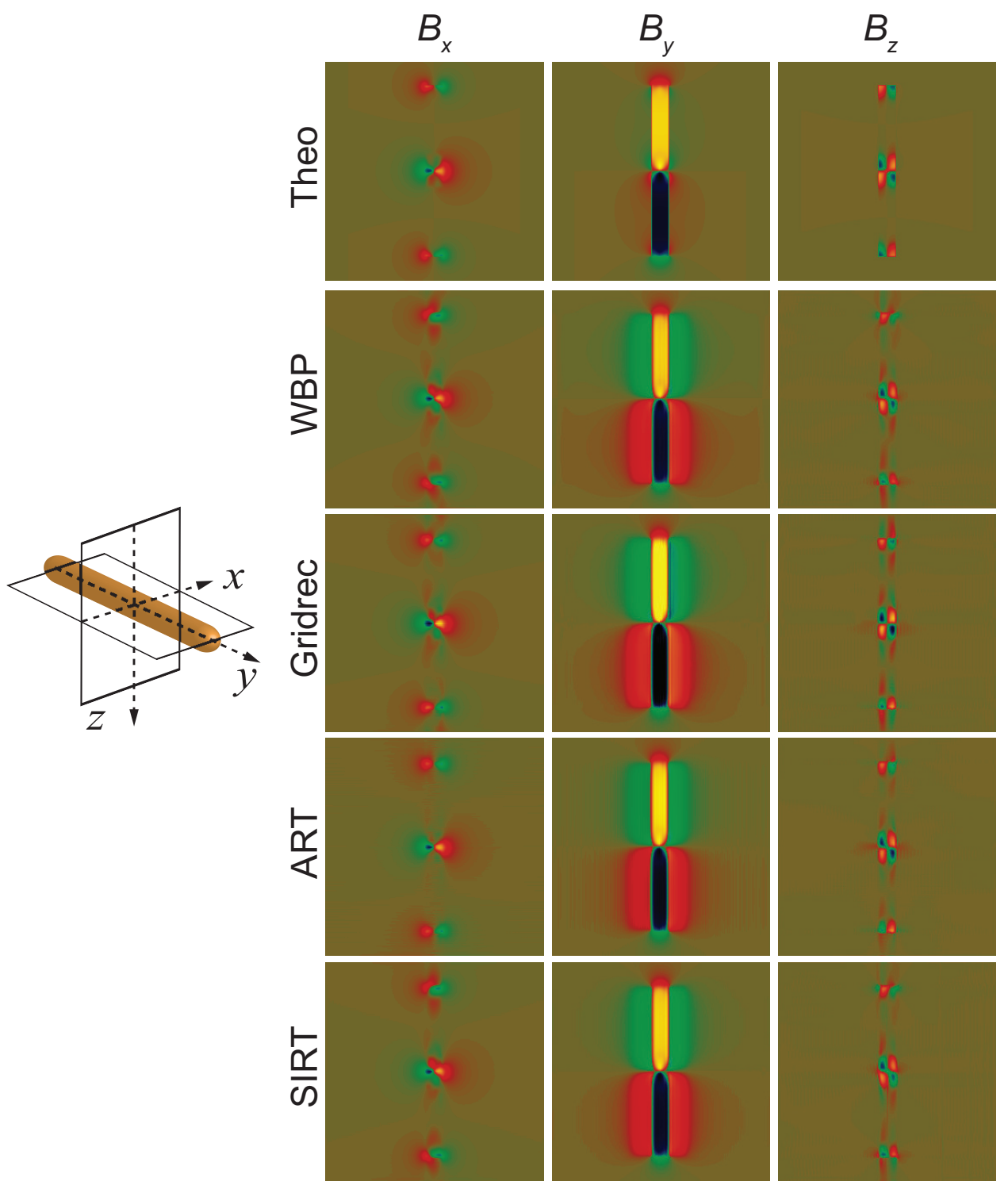

Figure 9: Comparison of reconstructed components of the vector field shown as 2D images along the $x-y$ plane. The orientation of the planes and the nanowire is indicated in the schematic. All the images are scaled to the same intensity range indicated by the color bar. 


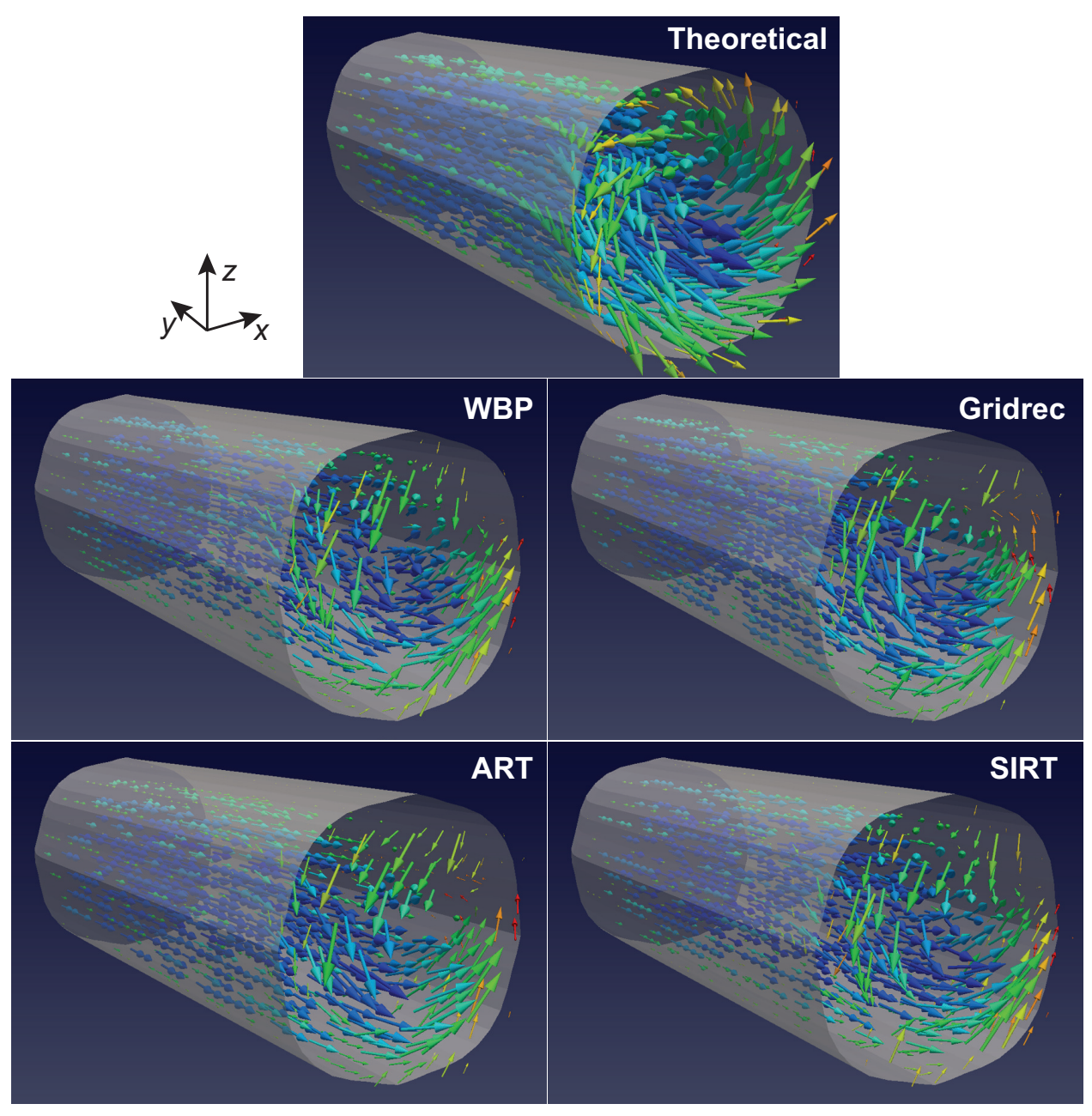

Figure 10: 3D vector field plots comparing the reconstructed magnetization in the nanowire for different methods. The data is clipped along the $x-z$ plane at $y=2$ for clarity. 